\title{
Extra Mixing in Globular Clusters of Varying Metallicity
}

\author{
George C. Angelou* \\ Centre for Stellar and Planetary Astrophysics, School of Mathematical Sciences, Monash \\ University, Melbourne, VIC 3800, Australia. \\ E-mail: george.angeloudmonash.edu
}

Sarah L. Martell

Australian Astronomical Observatory, North Ryde, NSW 2113, Australia

\section{Ross P. Church}

Lund Observatory, Department of Astronomy and Theoretical Physics, Box 43, SE-221 00 Lund,

Sweden.

\section{Richard J. Stancliffe}

Argelander-Institut für Astronomie, Auf dem Hügel 71, D-53121 Bonn, Germany

\section{John C. Lattanzio}

Centre for Stellar and Planetary Astrophysics, School of Mathematical Sciences, Monash University, Melbourne, VIC 3800, Australia.

\begin{abstract}
Canonical stellar evolution predicts that first dredge-up (FDU) is the only means by which red giant branch (RGB) stars change their surface abundances. However, observations of low-mass RGB stars at essentially all metallicities display trends in $\mathrm{C}, \mathrm{N}$ and Li that cannot be accounted for by the FDU event. Some 'extra mixing' over a timescale longer than that of FDU is responsible for cycling the products of partial hydrogen burning into the convective envelope. That $\mathrm{C}, \mathrm{N}$ and Li display clear variation with magnitude suggests that the changes are occurring in situ and not a result of earlier pollution. We focus on the evolution of $[\mathrm{C} / \mathrm{Fe}]$ in globular clusters across a large metallicity range $([\mathrm{Fe} / \mathrm{H}) \approx-2.2$ to -1.1$)$ to probe this extra mixing. Observations are compared to our stellar models that incorporate a $1 \mathrm{D}$ diffusive thermohaline mixing approximation. After taking into account the various populations within globular clusters, we find that thermohaline mixing can qualitatively reproduce the observed abundance trends with metallicity. However, the models do not always match the carbon abundance at the tip of the RGB in the most metal-poor clusters. Whether this is a failure of the models is unclear because the stars appear to conflict with standard evolution also. We require homogeneous data to confirm the result.
\end{abstract}

XII International Symposium on Nuclei in the Cosmos

August 5-12, 2012

Cairns, Australia

\footnotetext{
* Speaker.
} 


\section{Introduction}

Although it is now well understood that globular clusters (GCs) are not simple stellar populations, they still possess properties that make them useful diagnostics of stellar models. The number of stars in these systems provide a populated colour-magnitude diagram, and allow the determination of distances and ages. As they traverse the Galaxy, the stars within are gravitationally bound and unless perturbed, remain in the environment in which they were born. Thus GCs are preferable to collections of field stars that may contain objects that vary in age, composition, distance, origin and whose parameters are less certain. Although it is true that GCs are not entirely homogeneous, it is believed that they are comprised of distinct and identifiable populations which are homogeneous. Photometric studies suggest that some clusters display splitting in the HR diagram at various evolutionary stages [ [R8, [2] . A second population enriched in ${ }^{4} \mathrm{He}$ is required in some clusters as an explanation for the horizontal branch morphology [Q]. One further piece of evidence comes in the form of a dichotomy in the $\mathrm{CN}$ band strength. The $\mathrm{CN}$ band strength is an indicator of nitrogen content in stars. For archetypal clusters the favoured scenario is that ejecta from a fraction of the first generation of stars (initially $\mathrm{C}-\mathrm{O}-\mathrm{Mg}$ rich, with the same chemical composition as field stars of the same metallicity) mix with primordial gas, providing a medium from which a second generation (C-O-Mg poor and $\mathrm{N}-\mathrm{Na}-\mathrm{Al}$ rich) form. New stars that form from the polluted gas, which has undergone full $\mathrm{CNO}$ cycling, are inherently enriched in nitrogen as well as ${ }^{4} \mathrm{He}$ compared to the primordial generation [25]. Pollution from a progenitor that has undergone advanced hydrogen burning is also an explanation for the the presence of the well known $\mathrm{O}-\mathrm{Na}$ and $\mathrm{Mg}-\mathrm{Al}$ anticorrelations [ए]].

In addition, observations of GC stars have indicated that some of these light elements vary in situ as the stars ascend the RGB. The first change to the stellar surface abundance is attributed to FDU [20]. Mixing is associated with the inwards migration of the base of the convective envelope which brings to the surface the products of partial hydrogen burning. Depletion in carbon is accompanied by enhancement in nitrogen until the convective envelope recedes outwards and these changes are brought to a halt. These modest changes do not significantly alter the $\mathrm{CN}$ band strength and thus do not obnubilate the multiple population picture.

Studies have also shown a clear distinction between the abundances of stars below the GC luminosity function bump (LF bump) and those above [33, [30, 23]]. The onset of this so called 'extra mixing' is thus thought to be related to the hydrogen burning shell erasing the composition discontinuity left behind by FDU. As the name suggests such mixing is not predicted by canonical stellar evolution although it is understood from observations that the mixing must occur over a range of masses and metallicities ([32] and references therein). The mixing appears active in giants of all metallicities from solar to at least $[\mathrm{Fe} / \mathrm{H}] \approx-2.5$ [15] and masses less than $\approx 2.5 M_{\odot}$ [22], although not necessarily with equal efficiency throughout these mass and metallicity ranges. In fact, $[$ [, 0$]$ amongst others demonstrate that the mixing is more efficient at low metallicity. A form of non-convective mixing seems responsible whereby the products of partial hydrogen burning are cycled into the convective envelope, and conversely envelope material is transported to regions near the burning shell where it can be processed. That the abundance variations display a correlation with luminosity suggests that the mixing is occuring in situ and not due to pollution from a previous generation (see the review of [ए7] on multiple populations in GCs). 
The cause of the extra mixing is still unresolved but possible candidate mechanisms include: rotational mixing [35, [1, [26], magnetic fields [27, [1, [1]], internal gravity waves ([ए]]) and ther-

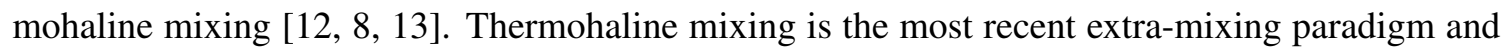
serves as the focus of this study. Mixing is thought to arise when material of higher molecular weight $(\mu)$ sits upon that of lower $\mu$. Such an inversion is not usually found in stellar interiors but may develop following FDU due to the reaction ${ }^{3} \mathrm{He}\left({ }^{3} \mathrm{He}, 2 \mathrm{p}\right){ }^{4} \mathrm{He}$. Note that although this is a fusion reaction it produces more particles than it consumes. It is one of the first reactions to occur in regions that undergo hydrogen burning but any effect on $\mu$ is usually swamped by reactions that raise $\mu$. When the hydrogen shell approaches the homogenised zone left behind by FDU, the ${ }^{3} \mathrm{He}$ reaction can dominate and without a previous $\mu$ gradient present, an inversion can develop.

In this study we model stars at three different metallicities, with the effects of thermohaline mixing included. We then compare these models to observations of GCs. Our results are based on that of [四, ㅁ] where the clusters NGC $5272(\mathrm{M} 3)$ and NGC $6205(\mathrm{M} 13)([\mathrm{Fe} / \mathrm{H}] \approx-1.5)$ and NGC 7078 (M15), NGC 6341 (M92) and NGC $5466([\mathrm{Fe} / \mathrm{H}] \approx-2.2)$ were all explored. We also present new data for the cluster NGC $6171([\mathrm{Fe} / \mathrm{H}] \approx-1.1)$.

\section{Method}

We use MONSTAR (the Monash version of the Mt. Stromlo evolution code; see [四]) to produce stellar models for the clusters. Our results are based on that of [U, [1] where thermohaline mixing is modelled as a diffusive process with coefficient of:

$$
D_{t}=C_{t} K\left(\frac{\varphi}{\delta}\right) \frac{-\nabla_{\mu}}{\left(\nabla_{\mathrm{ad}}-\nabla\right)} \quad \text { for } \nabla_{\mu}<0
$$

where $\varphi=(\partial \ln \rho / \partial \ln \mu)_{P, T}, \delta=-(\partial \ln \rho / \partial \ln T)_{P, \mu}, \nabla_{\mu}=(\partial \ln \mu / \partial \ln P), \nabla_{\mathrm{ad}}=(\partial \ln T / \partial \ln P)_{\mathrm{ad}}$, $\nabla=(\partial \ln T / \partial \ln P)$ and $K$ is the thermal diffusivity. The theory predicts a mixing depth from the nuclear burning thus $C_{t}$ sets the mixing speed and acts as our dimensionless free parameter. The formalism is based on that developed by [B] ] and [ [2]] and advocated by [ [8]. Our set of reaction rates for hydrogen burning come primarily from [14]] and [18]]. For the ${ }^{14} \mathrm{~N}(\mathrm{p}, \gamma){ }^{15} \mathrm{O}$ reaction an updated rate from Champagne (2004, private communication) is used which is very similar to the recent LUNA rate. This rate is a factor of two less than previously thought.

We have compiled observational data for globular clusters over a range of metallicities. Data are sourced from the literature (see [[]]) as well as our own observations (NGC 6171). The data are compared to stellar evolution models with thermohaline mixing. The mixing efficiency is kept constant in all cases and is based on what we believe to be the best fit to the observations $\left(C_{t}=1000\right.$, see also [ $[\mathrm{B}]$ and [34]). Note that the efficiency we have chosen is higher than implied by recent 3D modelling of the process [ए]] but is consistent with the theoretical derivation of [B]].

\section{Results}

In Figure 1 we have grouped clusters according to metallicity. Plotted are $[\mathrm{C} / \mathrm{Fe}]$ abundances for giants in each GC as a function of absolute visual magnitude. Observational data for each clus-

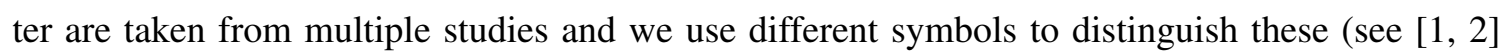



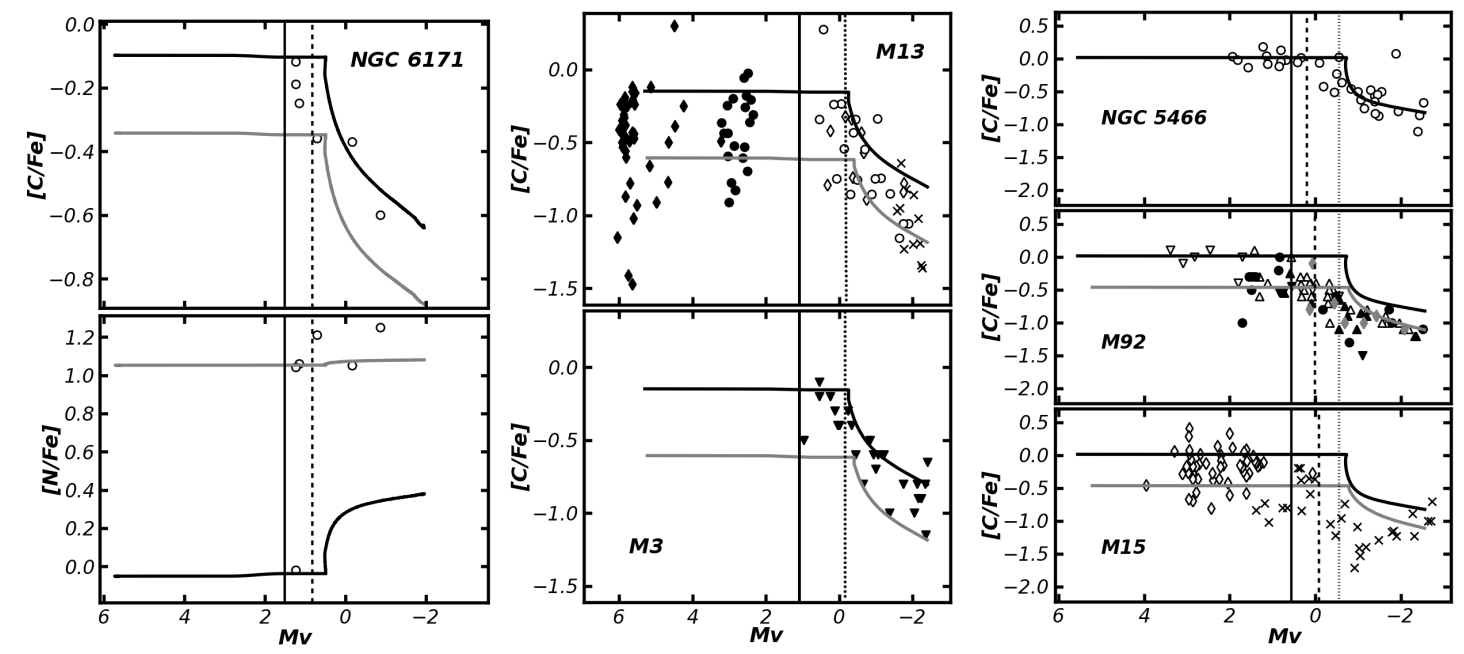

Figure 1: $[\mathrm{C} / \mathrm{Fe}]$ and $[\mathrm{N} / \mathrm{Fe}]$ abundances as a function of absolute magnitude for giants in the specified globular clusters. The various symbols denote data from the respective cluster surveys utilised. The sources of these studies can be found in [四, []. In all panels the solid vertical line denotes the end of first dredge-up and broken lines represent observational determinations of the LF bump ([24]). The black and grey curves are our stellar models with thermohaline mixing that try to simultaneously account for pre- and post- LF bump abundances. The initial abundances for each curve are selected to try to account for the presence of multiple populations in each cluster.

for sources). The black and grey curves represent the evolutionary calculations with thermohaline mixing. At each metallicity the two curves differ only in their initial abundances. Through measurements of the $\mathrm{CN}$ bands in $\mathrm{M} 3$, the initial compositions of the distinct populations can be inferred. The two models at intermediate metallicity were chosen with initial abundances to reflect these data. In two of the low-metallicity clusters and in NGC 6171 models are provided simply to match the spread of the (expected) subgiant stars. The leftmost panels, and our newest result, is for NGC $6171([\mathrm{Fe} / \mathrm{H}] \approx-1.1)$ where we also provide $[\mathrm{N} / \mathrm{Fe}]$ abundances as a function of magnitude. We will soon report on the Keck/LRIS spectra that these abundances were derived from (Martell et al. 2013, in prep) but here we present the results for six stars. The results of our initial models for this cluster are ambiguous and we require more data before drawing further conclusions. The two middle panels are for the intermediate-metallicity clusters $\mathrm{M} 3$ and $\mathrm{M} 13([\mathrm{Fe} / \mathrm{H}] \approx-1.5)$. Our models are able to explain most stars in the intermediate-metallicity clusters save for the most $[\mathrm{C} / \mathrm{Fe}]$ depleted subgiants in M13. It appears as if these most carbon-depleted subgiants lack counterparts on the RGB. This could be the role of a small sample size or the effect of systematic offsets from different data sources. Note these stars are faint and may also have large associated errors.

The final three panels are for the metal-poor GCs NGC 5466, M92 and M15, and perhaps are the most interesting. Although [ 3$]$ find evidence for the presence of multiple populations in NGC 5466, we require only a single model to explain the carbon depletion. The abundance differences between the two populations do not appear as significant as seen in other clusters. The more massive counterparts, M92 and M15, appear to deplete more carbon than predicted by the models. Of greater concern is the fact that depletion seems to be occurring before the theoretical limit predicted by standard evolution. The black line indicates the end of FDU whilst the broken 
lines are two estimates of the luminosity function bump; the expected onset of extra mixing. Mixing appears to begin between these magnitudes. Either utilising data from multiple studies carried out with different instruments is affecting our interpretation or our understanding of stellar evolution at low metallicity is seriously wrong. In [[] we investigated the possibility of deeper FDU and found this to be an unsatisfactory solution as FDU would need to be deeper in M92 and M15 but not NGC 5466. Homogeneous data (i.e., observations carried out with a single instrument) over a large range in luminosity will help with this mystery.

\section{Summary}

We have implemented an extra-mixing mechanism that requires only one free parameter $\left(C_{t}\right)$ and tested this across GCs of varying metallicity. In each case we selected a best fit initial abundance to account for the presence of multiple populations. An empirically based mixing speed was used and the mixing depth was dictated by the metallicity and nuclear burning. Keeping the $C_{t}$ value constant across our cluster sample has yielded mixed results. Although our models can, for the most part, account for stars in the intermediate-metallicity clustersit is at low metallicity that issues arise. Our models cannot explain the level of depletion seen in M92 and M15. Furthermore, mixing appears to begin earlier than current stellar evolution theory predicts. If mixing begins before the bump, then it must remove the abundance discontinuity that itself is responsible for the bump. These clusters are not only a problem for thermohaline mixing but for stellar physics in general. A homogenous study of these clusters that focuses on $[\mathrm{C} / \mathrm{Fe}],[\mathrm{N} / \mathrm{Fe}]$ and $\mathrm{Li}$ over a large luminosity range would help identify the onset of mixing.

It is of course possible that thermohaline mixing does not govern the surface composition of low mass giants but this still does not help with the problem of mixing in M92 and M15. Recent 3D models of the process [ए]] and [36] suggest our efficiency is too high (see also the discussion by Stancliffe in this volume). Some studies also suggest that horizontal turbulence inhibits this mixing [2]]. As was the situation 40 years ago, there is still much work to be done in order to understand transport processes inside stars.

\section{References}

[1] Angelou, G. C., Church, R. P., Stancliffe, R. J., Lattanzio, J. C. and Smith, G. H. 2011, ApJ, 728, 79

[2] Angelou, G. C., Stancliffe, R. J., Church, R. P., Lattanzio, J. C. and Smith, G. H. 2012, ApJ, 749, 128

[3] Busso, M., Wasserburg, G. J. and Nollett, K. M. and Calandra, A. 2007, ApJ, 671, 802

[4] Campbell, S. W. and Lattanzio, J. C. 2008, A\&A, 490, 769

[5] Chanamé, J., Pinsonneault, M. and Terndrup, D. M. 2005, ApJ, 631, 540

[6] Charbonnel, C. 1994, A\&A, 282, 811

[7] Charbonnel, C. 1996, ASPC, 98, 213

[8] Charbonnel, C., and Zahn, J. 2007, A\&A, 476, L29

[9] D'Antona, F. and Caloi, V. 2008, MNRAS, 390, 693

[10] Denissenkov, P. A. and Merryfield, W. J. 2011 ApJL, 727, L8 
[11] Denissenkov, P. A. and Tout, C. A. 2000, MNRAS, 316, 395

[12] Eggleton P. P, Dearborn D. S. P., and Lattanzio J. L. 2006, Science, 314, 1580

[13] Eggleton P. P, Dearborn D. S. P., and Lattanzio J. L. 2008, ApJ, 677, 581

[14] Fowler, W. A., Caughlan, G. R. and Zimmerman, B. A. 1975, Ann.Rev., 13, 69

[15] Gratton, R. G., Sneden, C., Carretta, E., and Bragaglia, A. 2000, A\&A, 354, 169

[16] Gratton, R. G., Bonifacio, P., Bragaglia, A., Carretta, E., Castellani, V., Centurion, M., Chieffi, A., Claudi, R., Clementini, G., D’Antona, F, Desidera, S., François, P., Grundahl, F., Lucatello, S., Molaro, P., Pasquini, L., Sneden, C., Spite, F. and Straniero, O. 2001, A\&A, 369, 87

[17] Gratton, R. G., Carretta, E. and Bragaglia, A. 2012, A\&A Rev, 20, 50

[18] Harris, M. J., Fowler, W. A., Caughlan, G. R. and Zimmerman, B. A. 1983, Ann.Rev., 21, 165

[19] Hubbard, E. N. and Dearborn, D. S. P. 1980, ApJ, 239, 248

[20] Iben, I, Jr. 1967, ApJ, 147, 624

[21] Kippenhahn, R., Ruschenplatt, G., and Thomas, H. 1980, A\&A, 91, 175

[22] Lambert, D. L. and Ries. 1977, ApJ, 217, 508

[23] Martell, S. L., Smith, G. H. and Briley, M. M. 2008 AJ, 136, 2522

[24] Nataf, D. M., Gould, A. P. and Pinsonneault, M. H. and Udalski, A. 2011 arXiv: http://adsabs.harvard.edu/abs/2011arXiv1109.2118N

[25] Norris, J., Cottrell, P. L., Freeman, K. C. and Da Costa, G. S. 1981, Ap.J., 244, 205

[26] Palacios, A., Charbonnel, C. and Talon, S. and Siess, L. 2006, A\&A, 453, 261

[27] Palmerini, S., Busso, M., Maiorca, E. and Guandalini, R. 2009, PASA, 26, 161

[28] Piotto, G., Bedin, L. R., Anderson, J., King, I. R., Cassisi, S., Milone, A. P., Villanova, S., Pietrinferni, A. and Renzini, A. 2007, ApJL, 661, L53

[29] Piotto, G., Milone, A. P., Anderson, J., Bedin, L. R., Bellini, A., Cassisi, S.,Marino, A. F., Aparicio, A. and Nascimbeni, V. 2012, arXiv: http://adsabs.harvard.edu/abs/2012arXiv1208.1873P

[30] Shetrone, M. 2003, ApJL, 585, L45

[31] Shetrone, M., Martell, S. L., Wilkerson, R., Adams, J., Siegel, M. H., Smith, G. H. and Bond, H. E. 2010, AJ, 140, 1119

[32] Smiljanic, R., Gauderon, R., North, P., Barbuy, B., Charbonnel, C. and Mowlavi, N. 2009, A\&A, 502, 267

[33] Smith, G. H. and Martell, S. L. 2003, PASP, 115, 1211

[34] Stancliffe, R. J., Church, R. P., Angelou, G. C. and Lattanzio, J. C. 2009, MNRAS, 396, 2313

[35] Sweigart, A. V. and Mengel, J. G. 1979, ApJ, 229, 624

[36] Traxler, A. and Garaud, P. and Stellmach, S. 2011, ApJL, 728, L29

[37] Ulrich, R. K. 1972, ApJ, 172, 165 